\title{
BMJ Open A pilot randomised controlled trial of a periodised resistance training and protein supplementation intervention in prostate cancer survivors on androgen deprivation therapy
}

\author{
Jacqueline L Kiwata, ${ }^{1}$ Tanya B Dorff, ${ }^{2}$ E Todd Schroeder, ${ }^{1}$ George J Salem, ${ }^{1}$ \\ Christianne J Lane, ${ }^{3}$ Judd C Rice, ${ }^{4}$ Mitchell E Gross, ${ }^{5}$ Christina M Dieli-Conwright ${ }^{1,6}$
}

To cite: Kiwata JL, Dorff TB, Todd Schroeder E, et al. A pilot randomised controlled trial of a periodised resistance training and protein supplementation intervention in prostate cancer survivors on androgen deprivation therapy. BMJ Open 2017;7:e016910. doi:10.1136/ bmjopen-2017-016910

- Prepublication history for this paper is available online. To view these files please visit the journal online (http://dx.doi org/10.1136/bmjopen-2017016910)

Received 20 March 2017 Revised 7 June 2017 Accepted 9 June 2017

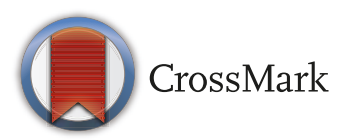

For numbered affiliations see end of article.

Correspondence to

Jacqueline L Kiwata; kiwata@ usc.edu

\section{ABSTRACT}

Introduction Prostate cancer survivors (PCS) receiving androgen deprivation therapy (ADT) experience deleterious side effects such as unfavourable changes in cardiometabolic factors that lead to sarcopenic obesity and metabolic syndrome (MetS). While loss of lean body mass (LBM) compromises muscular strength and quality of life, MetS increases the risk of cardiovascular disease and may influence cancer recurrence. Exercise can improve LBM and strength, and may serve as an alternative to the pharmacological management of MetS in PCS on ADT. Prior exercise interventions in PCS on ADT have been effective at enhancing strength, but only marginally effective at enhancing body composition and ameliorating cardiometabolic risk factors. This pilot trial aims to improve on existing interventions by employing periodised resistance training (RT) to counter sarcopenic obesity in PCS on ADT. Secondary aims compare intervention effects on cardiometabolic, physical function, quality of life and molecular skeletal muscle changes. An exploratory aim examines if protein supplementation (PS) in combination with RT elicits greater changes in these outcomes.

Methods and analysis A $2 \times 2$ experimental design is used in 32 PCS on ADT across a 12-week intervention period. Participants are randomised to resistance training and protein supplementation (RTPS), RT, PS or control. RT and RTPS groups perform supervised RT three times per week for 12 weeks, while PS and RTPS groups receive $50 \mathrm{~g}$ whey protein per day. This pilot intervention applies a multilayered approach to ameliorate detrimental cardiometabolic effects of ADT while investigating molecular mechanisms underlying skeletal muscle changes in PCS.

Ethics and dissemination This trial was approved by the University of Southern California Institutional Review Board (HS-13-00315). Results from this trial will be communicated in peer-reviewed publications and scientific presentations.

Trial registration number NCT01909440; Pre-results.

\section{INTRODUCTION}

The number of prostate cancer survivors (PCS) is steadily growing, with an estimated
Strengths and limitations of this study

- A strength of this study is the use of a detailed periodisation model as a structured form of resistance training progression to optimise improvements in body composition and physical function.

- This study also investigates molecular regulators of skeletal muscle remodelling to define mechanisms underlying a resistance exercise intervention in prostate cancer survivors.

- An innovative aspect is the coupling of protein supplementation with resistance exercise to maximise the potential for lean mass increases. However, neither diet or caloric intake is controlled.

- Because of the limited number of patients allocated across four groups, results from this study offer preliminary insight and hypothesis-generating data for larger trials.

3 million survivors in the USA in 2015 and a 5-year survival rate of nearly $99 \%{ }^{1}$ An important component of prostate cancer therapeutics is androgen deprivation therapy (ADT), which reduces endogenous testosterone production to castrate levels in an effort to impede testosterone-driven tumour progression. The use of ADT has increased such that it has become the mainstay of treatment for locally advanced or metastatic prostate cancer with approximately $45 \%$ of PCS receiving ADT during the first year after diagnosis. ${ }^{2}$

Despite important clinical benefits such as improvement in survival, ADT is associated with numerous detrimental effects. Due to marked reductions in testosterone, PCS on ADT lose lean body mass (LBM) and gain fat mass, a condition known as sarcopenic obesity, and experience adverse changes 
to cardiovascular risk factors associated with metabolic syndrome (MetS). ${ }^{2}$ MetS comprised a constellation of cardiometabolic variables, including hypertension, central adiposity, hypertriglyceridaemia, hyperglycaemia and low high-density lipoprotein cholesterol (HDL-C), with insulin resistance as the underlying feature. ${ }^{3}$ As MetS is an established risk factor for cardiovascular disease, and MetS-related biomarkers such as insulin and insulin-like growth factor-1 (IGF-1) are implicated in prostate tumorigenesis, ${ }^{4}$ interventions targeting MetS components may reduce the risk of cardiovascular disease comorbidity in PCS as well as slow disease progression. Furthermore, patients receiving ADT lose approximately 2\%-4\% LBM in the first year of therapy, and compared with healthy, age-matched men, PCS on ADT exhibit significantly lower physical function (ie, muscle strength, balance) and health-related quality of life. ${ }^{5}$ Ultimately, declines in muscle strength contribute to fall risk and physical function impairment, which may impact the cost of continuing care, exacerbating prostate cancer-related lifetime spending beyond current estimates of $\$ 4.0$ billion. ${ }^{6}$ Thus, an intervention that ameliorates sarcopenic obesity, attenuates MetS and restores physical function can serve as a crucial aspect of long-term care for PCS.

Treatment of MetS primarily consists of the pharmacological management of the individual components of MetS, such as the targeting of dyslipidemia through statins, hyperglycaemia through metformin or hypertension through antihypertensive therapy. ${ }^{3}$ However, these pharmaceutical approaches do not comprehensively address the diverse, adverse alterations due to ADT. Because lifestyle factors such as physical activity level can affect the risk of MetS, and exercise can directly target side effects of ADT, employing exercise as a therapeutic option may be a useful alternative to pharmacological approaches for PCS on ADT. There is much support for improving cardiometabolic variables and physical function through exercise in older adults and patients with type 2 diabetes ${ }^{7}$; however, less evidence exists for the efficacy of exercise training in PCS on ADT. The few randomised controlled trials with exercise interventions conducted in PCS have been effective at enhancing muscular strength, ${ }^{8-13}$ but only marginally effective at ameliorating cardiometabolic risk factors, ${ }^{91014-16}$ reducing fat mass ${ }^{91517}$ and increasing LBM. ${ }^{112}$ Among those studies specifically targeting MetS criteria in PCS, none have reported a reduced prevalence of MetS as a result of exercise. ${ }^{9}{ }^{10}{ }^{14-16}$ Improvements in individual MetS variables in PCS on ADT have been observed following a combined programme of resistance training and aerobic training (RT+AT), ${ }^{9}{ }^{10}$ or a regimen of high-intensity interval training. ${ }^{15}$ Furthermore, in studies investigating LBM changes in PCS on $\mathrm{ADT}, \mathrm{RT}+\mathrm{AT}$ has been observed to attenuate LBM loss or elicit moderate increases in LBM (range: 0\%-2.7\%) with concomitant improvements in strength. ${ }^{11}$ Thus, exercise may be an effective intervention to counter adverse cardiometabolic and physical function alterations in PCS on ADT, but refinements to existing training methodologies are necessary to optimally improve health and fitness outcomes.

Although most of the previously conducted interventions in PCS on ADT have met the American College of Sports Medicine/American Cancer Society's (ACSM/ ACS) recommendation for exercise in cancer survivors, ${ }^{1819}$ studies that were most successful in favourably altering LBM or MetS components incorporated progressive intensity or volume. Yet, only a few exercise interventions in PCS on ADT have employed periodisation, ${ }^{20} 21$ defined as the systematic progression of the acute programme variables of load, volume and rest period over the course of the training duration. Used primarily in athletic populations, periodisation is regarded as a superior method for optimally eliciting physiological and performance-related improvements. ${ }^{22}$ Periodised resistance training (RT) may represent an optimal form of exercise in simultaneously countering cardiometabolic, body composition and physical function alterations as periodised interventions in insulin-resistant individuals have demonstrated significant improvements in all outcomes. ${ }^{23}{ }^{24}$ In addition, periodisation can be used to mitigate fatigue,$^{25}$ a prevalent side effect in many types of cancer treatment. Despite these purported advantages, the use of periodisation in therapeutic exercise interventions for cancer survivors has been underexploited, particularly in countering the metabolic side effects of ADT.

An additional issue is that even in the absence of ADT and disease healthy older adults exhibit a blunted response to anabolic stimuli compared with young adults. A single bout of resistance exercise has been shown to stimulate robust muscle protein synthesis in young adults 4-24hours after exercise, while the same exercise bout performed by older adults evokes small, usually non-significant, increases in protein synthesis. ${ }^{26}$ This blunted response has been suggested to result in a reduced hypertrophic response over a long-term programme of RT. To augment protein synthesis in older adults, the combined use of RT and milk-derived protein supplementation (PS) has been proposed as a strategic method for eliciting muscle hypertrophy. Although numerous investigations support the use of postexercise PS to enhance acute muscle protein synthesis, ${ }^{27}$ limited data attest to the longterm effects of supplementation and RT on hypertrophy and strength gains in older adults. ${ }^{28}$ Nonetheless, the addition of PS to a periodised programme of RT may augment LBM increases in PCS on ADT beyond RT alone.

The blunted response to anabolic stimuli observed in older adults has been attributed to reductions in skeletal muscle protein synthesis, mediated through the IGF-1/protein kinase B (Akt)/mammalian target of rapamycin (mTOR) pathway, and increases in muscle protein degradation, characterised by persistent activation of the catabolic myostatin/activin pathway. ${ }^{29}$ Both IGF-1/Akt/mTOR and myostatin/activin pathways are responsive to exercise-related stimuli, with increases in IGF-1/Akt/mTOR and decreases in myostatin/activin observed after acute and chronic RT in men. ${ }^{30-32}$ Thus, 
an intervention that chronically increases signalling through the anabolic IGF-1/Akt/mTOR pathway and suppresses signalling through the catabolic myostatin/ activin pathway is purported to increase muscle protein synthesis, which may lead to hypertrophic adaptations in the long term.

\section{OBJECTIVES}

This ongoing pilot study was designed for the primary aim of comparing the effect of a 12-week supervised, periodised RT intervention on sarcopenic obesity in men with prostate cancer on current or previous adjuvant ADT. For the primary hypothesis, a periodised RT intervention is expected to attenuate sarcopenic obesity to a greater extent than no exercise. For an exploratory hypothesis, the greatest attenuation in sarcopenic obesity will be observed in resistance training and protein supplementation (RTPS), followed by RT alone, PS alone, then control. Secondary aims investigate the impact of the periodised RT intervention on (1) cardiometabolic markers, including the individual MetS components of blood pressure, central adiposity, triglycerides, glucose and HDL-C, and other biomarkers (ie, insulin, IGF-1); (2) physical function, including muscular strength, cardiorespiratory fitness and functional task performance; (3) skeletal muscle regulation, including gene and protein expression of factors regulating skeletal muscle growth and atrophy; and (4) health-related quality of life. As this study represents the first intervention using periodisation and PS in PCS, the safety and feasibility of such a programme is also assessed.

\section{METHODS AND ANALYSIS \\ Experimental design}

Study enrolment and data collection began in May 2014. Men with prostate cancer expected to remain hypogonadal (total testosterone $<50 \mathrm{ng} / \mathrm{dL}$ ) throughout the 12-week study duration due to current or previous ADT administered for at least 3 months were enrolled. The study flow and study visit timeline are presented in figures 1 and 2, respectively. After obtaining written consent, participants are randomised in a 1:1 allocation ratio to one of four study groups: (1) RTPS, (2) RT, (3) PS or (4) control, with end points assessed at baseline (T1, T2), midpoint (T3) and post intervention (T4, T5).

Participants in the RTPS and RT groups perform 12 weeks of RT supervised by a certified exercise specialist and progressed according to a periodisation model. Participants in the PS and control groups are asked to maintain their current level of activity. In addition, as a means of enhancing retention and compliance, PS and control participants are given a home-based stretching programme for the duration of the 12-week intervention period and are offered the exercise programme at the end of the study.

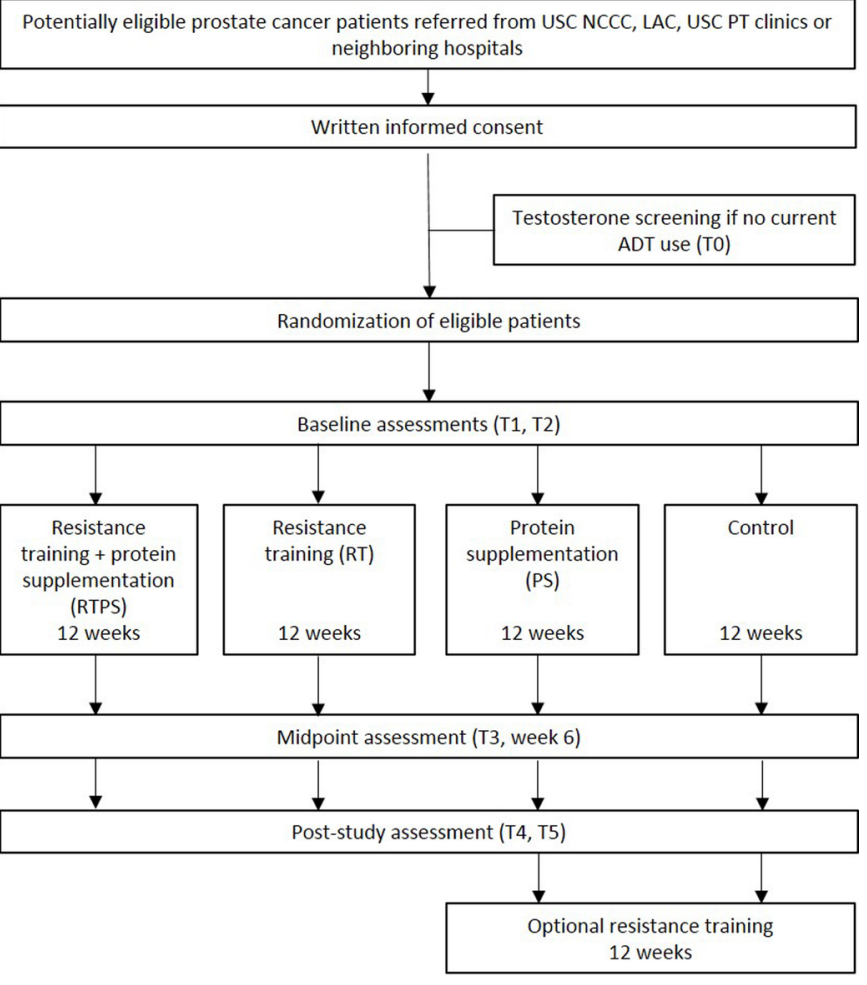

Figure 1 Study flow. ADT, androgen deprivation therapy; LAC, Los Angeles County Hospital; NCCC, Norris Comprehensive Cancer Center; PT, physical therapy; USC, University of Southern California.

\section{Study status}

Study enrolment has been completed, but the intervention and data collection (T4, T5) are ongoing.

\section{Participants}

Eligibility criteria

Men recruited for this study are diagnosed with prostate cancer, aged $\geq 50$, have no contraindications to exercise and are currently treated with gonadotropin-releasing hormone (GnRH) agonist/antagonist with or without antiandrogen for at least 12 weeks or have received prior treatment of GnRH agonist/antagonist and have serum testosterone concentration $<50 \mathrm{ng} / \mathrm{dL}$ at baseline and for the study duration. Metastatic patients are not excluded. Inclusion and exclusion criteria are provided in table 1.

\section{Recruitment strategy}

Patients are recruited by (1) referral from the medical oncology physicians and staff at the University of Southern California (USC) Norris Comprehensive Cancer Center (NCCC) or local hospitals; (2) referral from USC Physical Therapy clinics; (3) outreach at community survivorship meetings in the Los Angeles, California, area; and (4) advertisements in local newspapers. Interested patients are provided detailed information on the study protocol by the principal investigator, inclusion/exclusion criteria are verified and written consent is obtained. For patients not currently receiving $\mathrm{ADT}$ at the time of consent, an additional screening for testosterone is performed using the 


\begin{tabular}{|c|c|c|c|c|c|c|c|c|c|c|}
\hline \multicolumn{11}{|c|}{ STUDY DURATION } \\
\hline & \multicolumn{3}{|c|}{ Wk 0} & \multicolumn{4}{|c|}{ Wk $0-1$} & \multirow{2}{*}{$\begin{array}{c}\text { Wk } 2 \text { - } 13 \\
\begin{array}{c}12-\text { week } \\
\text { intervention }\end{array}\end{array}$} & \multirow{2}{*}{\multicolumn{2}{|c|}{$\begin{array}{c}\text { Wk } 14 \\
\text { Post-test }\end{array}$}} \\
\hline & Enrolment & \multicolumn{2}{|c|}{ Randomisation } & \multicolumn{4}{|c|}{ Pre-test } & & & \\
\hline TIMEPOINT & $-t_{1}$ & TO & 0 & $\mathrm{~T} 1$ & F1 & F2 & $\mathrm{T} 2$ & T3 & T4 & T5 \\
\hline Eligibility screen & $x$ & & & & & & & & & \\
\hline Informed consent & $x$ & & & & & & & & & \\
\hline Testosterone screen & & $x$ & & & & & & & & \\
\hline Randomisation & & & $x$ & & & & & & & \\
\hline Familiarisation & & & & & $x$ & $x$ & & & & \\
\hline INTERVENTIONS: & & & & & & & & & & \\
\hline Resistance Training & & & & & & & & $x$ & & \\
\hline $\begin{array}{r}\text { Protein } \\
\text { Supplementation }\end{array}$ & & & & & & & & $x$ & & \\
\hline Control Stretching & & & & & & & & $x$ & & \\
\hline ASSESSMENTS: & & & & & & & & & & \\
\hline Bodv comnosition* & & & & $x$ & & & & $x$ & $x$ & \\
\hline Blond drawe & & & & $x$ & & & & $x$ & $x$ & \\
\hline Muscle hionsy** & & & & $x$ & & & & & $x$ & \\
\hline Oulituof lifo & & & & $x$ & & & & & $x$ & \\
\hline Physical function & & & & & & & $x$ & $\mathrm{x}$ & & $X$ \\
\hline
\end{tabular}

Figure 2 Study visit timeline. *Primary end point; **optional assessment.

criteria of testosterone $<50 \mathrm{ng} / \mathrm{dL}$, with blood collected and analysed real time ( $\mathrm{T} 0$, figure 1 ). If total testosterone is found to be $50 \geq \mathrm{ng} / \mathrm{dL}$, the patient is considered ineligible for the study and dropped prior to randomisation. Testosterone concentration is again checked post intervention to ensure eligibility requirements have been met throughout the duration of the study.

\section{Interventions}

Participants in the RTPS and RT groups perform one-on-one RT three times per week for 12 weeks with an ACSM certified Cancer Exercise Trainer, with each session lasting approximately $45 \mathrm{~min}$. Participants in the PS and control groups perform a home-based flexibility programme three times per week for 12 weeks, with each session lasting approximately $5 \mathrm{~min}$.

\section{Resistance training programme (RTPS and RT groups)}

Participants in the RTPS and RT groups participate in a 12-week, supervised periodised RT programme at the USC Clinical Exercise Research Center (CERC) 3 days per week. At each session, the trainer documents attendance, and records soreness and fatigue from the prior session. Participants are allowed to make up missed sessions, extending the study period by an additional 2 weeks for a maximum duration of 14 weeks. Treatment will be discontinued if participants attend $<80 \%$ of the training sessions, miss more than four consecutive 
Table 1 Inclusion and exclusion criteria

\begin{tabular}{ll}
\hline Inclusion criteria & Exclusion criteria \\
\hline Males diagnosed with prostate cancer & Chemotherapy or radiation therapy within the past four weeks \\
$\geq 50$ years of age & Opioid-requiring cancer-related pain \\
Current treatment with ADT (GnRH agonist/antagonist & Acute coronary or vascular event within the past oneyear \\
with or without antiandrogen) for a minimum of 12 weeks & Major surgery within the past six months \\
OR Prior treatment with ADT and serum testosterone & Uncontrolled coronary heart disease \\
concentration $<50 \mathrm{ng} / \mathrm{dL}$ at baseline and for study duration & Neurological, orthopaedic or genitourinary limitations that \\
Permission from treating/study physician to participate in & preclude participation in exercise \\
exercise & History of allergic reaction or intolerance to whey protein \\
& (lactose intolerance is acceptable) \\
& Current use of N-acetylcysteine oro-lipoic acid supplements \\
& Current participation in a structured exercise programme
\end{tabular}

ADT, androgen deprivation therapy; $\mathrm{GnRH}$, gonadotropin-releasing hormone.

training sessions, or for patients not concurrently on ADT, total testosterone level $\geq 50 \mathrm{ng} / \mathrm{dL}$. In addition, a participant may be removed whenever he wishes.

The RT intervention consists of a systematically progressed resistance exercise programme focusing on hypertrophy with limited rest periods. Similar RT protocols have been shown to elicit improvements in strength, muscle thickness ${ }^{20}$ and appendicular lean mass ${ }^{21}$ in PCS on ADT, while improvements in the MetS components of waist circumference and HDL-C have been observed in overweight individuals. ${ }^{33}$ This intervention builds on these existing protocols by employing RT in the absence of aerobic training to emphasise changes in lean mass. In addition, this protocol incorporates structured, goal-oriented progression through explicitly defined mesocycles that advance load and repetition using a linear model of periodisation.

In the periodisation model, two mesocycles are used, with each week representing a microcycle (table 2). ${ }^{22}$ The first mesocycle consists of 4 weeks and begins with a preparation phase where training at intensities and repetitions in the muscular endurance range $(60 \%-67 \%$ 1RM) are performed. ${ }^{22} 34$ The purpose of this low-intensity, highvolume preparation phase is to establish a base of training and to increase tolerance of higher training intensities that occur later in the programme. These objectives are particularly important in a clinical population such as PCS, where safety is a major concern, and energy levels may be affected by treatment. ${ }^{18}$ The last week of the first mesocycle serves as a transition into a hypertrophy range, with training performed at $67 \% 1 \mathrm{RM}$. The second mesocycle consists of 8 weeks and progresses through a hypertrophy training range of $70 \%-83 \% 1 \mathrm{RM} .^{22}{ }^{34}$ Rest intervals are maintained at $1 \mathrm{~min}$ between sets and exercises to afford a moderate recovery of force production while sustaining metabolic stress in accordance with a hypertrophy training goal. ${ }^{35}$

Each 1-week microcycle includes three training days (table 3). One training session is focused on the lower extremities and trunk musculature, the second is focused on the upper extremities and trunk musculature, and the third session includes training of both upper and lower extremities. Dividing the weekly training volume in this manner allows each muscle group to be trained for a frequency of 2 times per week, affording greater recovery between heavy loading bouts. ${ }^{36}$ As recuperative ability is diminished in older men due to ADT and deconditioned training status, ${ }^{12}$ greater recovery between training sessions may optimise anabolic effects on muscle tissue and prevent overtraining.

The exercises selected for the intervention fall into one of two categories: (1) exercises that form the basis of the hypertrophy-specific routine and (2) dynamic movements that promote improvement in trunk stability. Trunk stabilisation exercises are purported to enhance sport performance and activities of daily living, ${ }^{37} 38$ as improvements in bench press and squat strength ${ }^{39}$ and ability to perform more difficult exercises ${ }^{40}$ were observed following a programme incorporating trunk stabilisation exercises. Hence, because the hypertrophy-specific routine in this study is progressive and consists of exercises that require postural stabilisation, trunk stabilisation movements are included.

The exercises are assigned a load according to the periodisation model intensity, with the exception of exercises targeting the trunk musculature, which are performed without external resistance. Each session begins with a dynamic warm-up consisting of exercises that emphasise trunk stabilisation and emulate the movements performed in the hypertrophy-specific routine (table 3). Exercises with minimal compression to the axial skeleton were selected for the hypertrophy-specific routine. However, in the event a participant is unable to perform an exercise due to pre-existing bone lesions or pain, an alternative exercise stimulating the same muscle group will be substituted and non-adherence to the original exercise programme is recorded. A dynamic warm-up is included rather than a static-stretching or aerobic warm-up because flexibility routines that consist of active, sport-specific exercises have been shown to positively influence performance outcomes following the warm-up bout. ${ }^{41}$ The session 
concludes with 5 min of static stretching as described in the flexibility protocol.

Flexibility protocol (RTPS and RT groups-supervised; PS and control groups-home-based)

The flexibility protocol consists of one set of 3-4 static stretching exercises held for $15 \mathrm{~s}$ and performed 3 days/ week. Because ACSM guidelines for cancer survivors recommend the stretching of major muscle groups on days that strength training exercises are performed, ${ }^{19}$ static stretching exercises are included at the end of every training session in the RTPS and RT groups. In an effort to reduce attrition in the PS and control groups, participants receive a home-based flexibility programme consisting of the same stretching exercises as the RTPS and RT groups, as performed in previous studies in PCS. ${ }^{42}$ Since the flexibility exercises are low intensity, low impact and low volume, performance of the stretching by the PS and control groups should not affect caloric expenditure or the targeted outcomes. In addition, no flexibility exercises are included that would be contraindicated for participants with axial skeletal bone metastases, including spinal flexion/extension/rotation. ${ }^{43}$ Muscle groups targeted in the stretching exercises are given in table 3. To increase compliance to the home-based programme, PS and control participants are instructed in performance of the flexibility exercises by the principal investigator as described in the 'Familiarisation' section, then are given a stretching band and a booklet detailing the stretching exercises to take home. In addition, PS and control participants complete weekly records of flexibility compliance and other exercises performed outside the study with a monetary compensation provided at midpoint and post intervention.

\section{Protein supplementation}

Participants in the RTPS or PS groups receive $50 \mathrm{~g} /$ day of whey protein isolate (EnergyFirst, Manhattan Beach, California, USA) for 12 weeks. The $50 \mathrm{~g}$ daily supplement is divided into two $25 \mathrm{~g}$ doses, with each $25 \mathrm{~g}$ dose containing $112.5 \mathrm{kcal}, 25 \mathrm{~g}$ protein (2 $\mathrm{g}$ leucine), $0 \mathrm{~g}$ fat and $3.75 \mathrm{~g}$ carbohydrate. A daily supplement of two $25 \mathrm{~g}$ doses is chosen to maximise stimulation of skeletal muscle protein synthesis as consumption of protein containing at least $2 \mathrm{~g}$ of the amino acid leucine was reported to increase protein synthesis in older adults to a similar extent as in young individuals. ${ }^{44}$ Participants are instructed to take doses separate from a meal in the morning and afternoon or evening. Participants are instructed to consume their regular food intake in addition to the supplements. For those participants in the RTPS group, one dose is given immediately after each RT session, as previous investigations suggest this is an optimal window for anabolic stimulus post exercise. ${ }^{45}$ Finally, participants record the date and time of protein ingestion in a log, which is collected on a weekly basis. PS will be discontinued if a participant experiences an intolerable adverse reaction to whey protein or if he wishes to stop the supplementation. 
Table 3 Sample training week in the hypertrophy mesocycle

\begin{tabular}{|c|c|c|c|c|c|c|c|c|}
\hline \multicolumn{3}{|l|}{ Lower/trunk } & \multicolumn{3}{|l|}{ Lower/upper } & \multicolumn{3}{|l|}{ Upper/trunk } \\
\hline Exercise & $\begin{array}{l}\text { Load } \\
\text { (RM, \%) }\end{array}$ & $\begin{array}{l}\text { Reps/ } \\
\text { sets }\end{array}$ & Exercise & $\begin{array}{l}\text { Load } \\
\text { (RM, \%) }\end{array}$ & $\begin{array}{l}\text { Reps/ } \\
\text { sets }\end{array}$ & Exercise & $\begin{array}{l}\text { Load } \\
\text { (RM, \%) }\end{array}$ & $\begin{array}{l}\text { Reps/ } \\
\text { sets }\end{array}$ \\
\hline \multicolumn{3}{|c|}{ Dynamic warm-up } & \multicolumn{3}{|c|}{ Dynamic warm-up } & \multicolumn{3}{|l|}{ Dynamic warm-up } \\
\hline Split squat & - & $15 \times 2$ & Air squat & - & $15 \times 2$ & Pushup & - & $15 \times 2$ \\
\hline Agility ladder & - & $\times 2$ & Pushup & - & $15 \times 2$ & Reverse pull-up & - & $15 \times 2$ \\
\hline Leg press & 70 & $10 \times 3$ & Leg press & 70 & $10 \times 3$ & Chest press & 70 & $10 \times 3$ \\
\hline Leg curl & 70 & $10 \times 3$ & Chest press & 70 & $10 \times 3$ & Lat pulldown & 70 & $10 \times 3$ \\
\hline \multirow[t]{2}{*}{ Leg extension } & 70 & $10 \times 3$ & Leg curl & 70 & $10 \times 3$ & Shoulder press & 70 & $10 \times 3$ \\
\hline & & & Lat pulldown & 70 & $10 \times 3$ & Seated row & 70 & $10 \times 3$ \\
\hline Hip bridge & - & $15 \times 3$ & Leg extension & 70 & $10 \times 3$ & Dead bug & - & $15 \times 3$ \\
\hline \multirow[t]{2}{*}{ Plank } & - & $30 s \times 3$ & Shoulder press & 70 & $10 \times 3$ & Plank & - & $30 s \times 3$ \\
\hline & & & Seated row & 70 & $10 \times 3$ & & & \\
\hline \multicolumn{3}{|l|}{ Flexibility } & \multicolumn{3}{|l|}{ Flexibility } & \multicolumn{3}{|l|}{ Flexibility } \\
\hline \multicolumn{2}{|c|}{ Hip flexors, extensors } & $15 \mathrm{~s} \times 1$ & \multicolumn{2}{|c|}{$\begin{array}{l}\text { Hip flexors, extensors } \\
\text { Shoulder extensors, flexors }\end{array}$} & $15 \mathrm{~s} \times 1$ & \multicolumn{2}{|c|}{ Shoulder extensors, flexors } & $15 s \times 1$ \\
\hline
\end{tabular}

$\mathrm{RM}$, repetition maximum.

In these scenarios, the participant will be allowed to continue the study in his original group allocation, while any missed doses of PS will be reported as non-compliance.

\section{Familiarisation}

Regardless of group randomisation, participants are instructed in proper technique, breathing and full range of motion on all resistance exercises used in strength testing prior to the baseline assessment (F1, F2; figure 2). The purpose of the familiarisation sessions is to increase the reliability of strength measurements. Familiarisation occurs over two separate sessions, with the same exercises performed during both sessions. Participants begin with a 5 min dynamic warm-up as described in the 'Resistance training programme' section, then complete the following exercises in the prescribed order: (1) leg press, (2) chest press, (3) leg curl, (4) seated shoulder press, (5) leg extension and (6) seated cable row. For each exercise, participants complete two sets of 10 repetitions at loads of approximately $20 \mathrm{RM}$ and $15 \mathrm{RM}$. The session concludes with static stretching exercises described in the 'Flexibility protocol' section. For the PS and control groups, participants are familiarised to the home-based stretching exercises by performing the stretches with a band under the supervision of the principal investigator.

\section{Outcomes}

All outcome measures assessed in this study are presented in table 4.

\section{Sarcopenic obesity}

The primary outcome is sarcopenic obesity, which is evaluated using measurements of appendicular skeletal mass (ASM) and body fat $\%$ at baseline (T1), midpoint (T3) and post intervention (T4) through whole-body dual-energy X-ray absorptiometry (DXA, Lunar GE iDXA, Fairfield, Connecticut, USA), following a 12 hours fast. Patient positioning and scanning is conducted as described by Hart $e t a l^{47}$ In-house coefficients of variation for DXA measurements calculated from a small subsample of patients with prostate cancer $(n=5)$ are $0.06 \%$ for ASM and $0.001 \%$ for body fat \%. Classification of sarcopenic obesity is made using the approach by Baumgartner, ${ }^{48}$ which was developed from reference data from the New Mexico Aging Process Study $(\mathrm{n}=301)^{49}$ and New Mexico Elder Health Survey $(\mathrm{n}=883)^{50}$ using GE/Lunar DXA, and used more recently in a cross-sectional analysis of sarcopenic obesity in data from the National Health and Nutrition Examination Survey $(\mathrm{n}=4984) .{ }^{51}$ Sarcopenia is defined as appendicular skeletal muscle index (ASMI $=\operatorname{ASM}(\mathrm{kg}) /$ height $\left.^{2}\left(\mathrm{~m}^{2}\right)\right)<7.26 \mathrm{~kg} / \mathrm{m}^{2}$, while obesity is defined as body mass index $>30 \mathrm{~kg} / \mathrm{m}^{2}$.

\section{Metabolic syndrome}

Participants are considered to present with MetS if criteria for three of five components of the syndrome are met $^{3}$ : (1) waist circumference $\geq 40$ inches, (2) triglycerides $\geq 150 \mathrm{mg} / \mathrm{dL}$ or treatment for this lipid abnormality, (3) HDL-C $\leq 40 \mathrm{mg} / \mathrm{dL}$ or treatment for this lipid abnormality, (4) systolic blood pressure $\geq 130 \mathrm{~mm} \mathrm{Hg}$ or diastolic blood pressure $\geq 85$ or treatment for hypertension and (5) glucose $\geq 100 \mathrm{mg} / \mathrm{dL}$ or previously diagnosed type 2 diabetes. In addition to analysing changes in each MetS component, changes in overall MetS status (yes/ no) and a combined score of the 5 MetS variables, calculated for each participant, are analysed from baseline to study completion.

\section{Venipuncture}

All serum markers are obtained from peripheral blood samples following a 12 hours fast at baseline, midpoint 


\begin{tabular}{|c|c|c|c|c|}
\hline Outcomes & Methods & T1, T2 & T3 & $\mathrm{T} 4, \mathrm{~T} 5$ \\
\hline \multicolumn{5}{|l|}{ Primary end point } \\
\hline \multicolumn{5}{|l|}{ Secondary end points } \\
\hline \multicolumn{5}{|l|}{ Components of MetS (5): } \\
\hline Blood pressure & Pressure cuff at rest & $x$ & $x$ & $x$ \\
\hline Waist circumference & Measuring tape & $x$ & $x$ & $x$ \\
\hline Cardiometabolic biomarkers & Analysed in peripheral blood & $x$ & $x$ & $x$ \\
\hline Muscle strength & $\begin{array}{l}\text { 10RM leg press, chest press, leg curl, shoulder } \\
\text { press, leg extension, seated row }\end{array}$ & $x$ & $x$ & $x$ \\
\hline Balance & $\mathrm{Y}$ balance test & $x$ & & $x$ \\
\hline \multicolumn{5}{|l|}{ Skeletal muscle studies } \\
\hline Gene expression & RT-PCR & $x$ & & $x$ \\
\hline Protein expression & SDS-PAGE, western blot & $x$ & & $x$ \\
\hline Quality of life & FACT-P, SF-36, BFI, CES-D & $x$ & & $x$ \\
\hline Feasibility & Accrual, retention, adherence & $x$ & & $x$ \\
\hline \multicolumn{5}{|l|}{ Additional end points } \\
\hline Prostate cancer history & $\begin{array}{l}\text { Family history, Gleason score, PSA history, } \\
\text { biopsy results }\end{array}$ & $x$ & & \\
\hline
\end{tabular}

BFI, Brief Fatigue Inventory; CES-D, Center for Epidemiologic Studies-Depression Scale; DXA, dual-energy X-ray absorptiometry; FACT-P, Functional Assessment of Cancer Therapy Prostate; HDL, high-density lipoprotein; LBM, lean body mass; MetS, metabolic syndrome; RM, repetition maximum; RT-PCR, real-time PCR; SF-36, Short Form-36; SDS-PAGE, sodium dodecyl sulfate polyacrylamide gel electrophoresis; PSA, prostate-specific antigen.

and post intervention. Blood is drawn from the antecubital or dorsal hand vein by phlebotomists at USC Keck hospital, processed within 2 hours, and stored at $-80^{\circ} \mathrm{C}$.

\section{Biomarkers}

In addition to the MetS biomarkers (glucose, HDL-C, triglycerides), the following cardiometabolic markers are obtained from peripheral blood samples as described above: (1) insulin, (2) IGF-1, (3) IGF binding protein, (4) haemoglobin A1c and (5) the inflammatory marker high-sensitivity $\mathrm{C}$ reactive protein. Lipids are analysed by the Norris Clinical Reference laboratory, while all other markers are analysed by the Diabetes and Obesity Research Institute at USC.

\section{Waist circumference}

Waist circumference is assessed by the same experienced investigator using a tape measure placed midway between the lower margin of the last palpable rib and the superior border of the iliac crest.

\section{Blood pressure}

At the baseline test, blood pressure is measured on both arms using an automated device (Welch Allyn, Skaneateles Falls, New York, USA) with the participant in the seated position following $5 \mathrm{~min}$ of quiet sitting. The arm with the higher blood pressure is recorded and used in subsequent measurements.

\section{Physical fitness}

All fitness measures are performed in the CERC by the same certified exercise trainer in the following order: (1) $400 \mathrm{~m}$ walk, (2) timed up and go, (3) stair climb, (4) balance and (5) muscular strength.

\section{Muscular strength}

Maximal voluntary strength is assessed at baseline, midpoint and at the completion of the intervention for the six exercises practised during the familiarisation sessions using 10RM strength tests. Participants complete a warm-up at an approximate 20RM load, which is 
estimated based on researcher experience and verbal questions pertaining to training history. Three attempts are given to reach the final 10RM load with a 2 min rest period between attempts. To determine load percentages used in the periodisation model, 1RM values are calculated from the 10RM. Specific regression equations for the leg press and chest press are used, with 1RM leg press $=1.2091 \quad(10 \mathrm{RM})+38.0908 \quad\left(\mathrm{R}^{2}=0.933\right),{ }^{52}$ and $1 \mathrm{RM}$ chest press $=(0.025 \times 10 \mathrm{RM} \times 10)+10 \mathrm{RM}\left(\mathrm{R}^{2}=0.99\right) .{ }^{53}$ For all other exercises, the equation $10 \mathrm{RM} / 0.75$ is used to calculate the $1 \mathrm{RM} .{ }^{54}$ Non-adherence is reported for participants unable to perform strength testing for a particular exercise due to pre-existing bone lesions or pain.

\section{Cardiorespiratory fitness}

The $400 \mathrm{~m}$ walk has been administered as a test of aerobic fitness in previous investigations in PCS on ADT. ${ }^{11}{ }^{12}$ Participants are instructed to walk as quickly as possible without running on a $200 \mathrm{~m}$ segment that is traversed twice. The investigator follows behind the participant for safety and to ensure the entire distance is traversed. Completion time is recorded to the nearest $01 \mathrm{~s}$.

\section{Functional power}

Previous investigations in PCS on ADT have used a stair climb as a test of functional power. ${ }^{11}{ }^{12}$ Participants are instructed to ascend a flight of 10 stairs one step at a time as quickly as possible without using a handrail. Timing begins when one foot steps on the third stair and ends when one foot reaches the ninth stair. Time is recorded to the nearest. $01 \mathrm{~s}$ using a stopwatch, and an average of three trials is calculated. One practice trial is given before the three trials.

\section{Mobility}

The timed up and go test reflects mobility and has been used to classify functional status of patients with cancer following treatment. ${ }^{55}$ Participants begin seated in a chair with hands on the armrests, are asked to rise, walk to a line on the floor $3 \mathrm{~m}$ from the chair, turn around and return to the same seated position as quickly and safely as possible. Timing is performed with a stopwatch, with score as the time to completion to the nearest. $01 \mathrm{~s}$. One practice trial given, and an average of time for three trials is calculated.

\section{Balance}

Participants perform the $\mathrm{Y}$ balance test (Functional Movement Systems, Danville, Virginia, USA), which has been shown to be a reliable measure of injury risk in patients with a variety of lower extremity conditions. ${ }^{56}$ Participants stand on one foot on a stance platform from which three pieces of polyvinylcholoride pipe, marked in $5 \mathrm{~mm}$ increments, extend in the anterior, posteromedial and posterolateral directions. Two investigators are positioned on either side of the participant during the test to ensure patient safety. The participant is instructed to push a target with the foot of the reaching limb in each of the three directions. Maximal reach is recorded for each foot, in each of the three directions, for a total of six measures. A practice trial is given for each foot in each direction. Participants unable to perform the balance test safely due to pre-existing bone lesions or pain are excluded, and non-adherence to the test is reported.

\section{Skeletal muscle cellular and molecular outcomes}

Molecular end points are selected to reflect signalling of various targets along the anabolic and catabolic pathways through gene and protein expression. The anabolic outcome measures include (1) peroxisome proliferator-activated receptor gamma (PPARG) coactivator 1 alpha (PGC-1 $\alpha 4$ ), a transcription coactivator associated with LBM maintenance in cachetic mice; (2) IGF-1; (3) phosphorylated Akt and (4) mTOR, all of which are myogenic factors that have been shown to be responsive to exercise. Catabolic measures include (1) myostatin, a negative regulator of myogenesis; (2) FOXO3a; (3) MAFbx and (4) MuRF1, all of which are factors involved in the ubiquitination and degradation of proteins.

\section{Muscle biopsy}

In order to understand the molecular mechanisms underlying potential LBM changes, muscle biopsies are performed as an optional procedure at the discretion of the participant before (T1) and after the intervention (T4). Using a previously described muscle biopsy procedure, ${ }^{57}$ a credentialed study physician obtains a percutaneous muscle sample $(150-200 \mathrm{mg}$ ) from the vastus lateralis. As resting levels of anabolic and proteolytic muscle regulators are desired, the biopsies occur after an 8 hour fast and between 72 and 96 hours following the exercise. ${ }^{58}$ Biopsy specimens are collected under local anaesthesia (1\% lidocaine) and sterile conditions using a $5 \mathrm{~mm}$ Stille muscle biopsy needle (Micrins Surgical, Lake Forest, Illinois, USA) from the midportion of the vastus lateralis muscle. The postintervention biopsy is performed at a distance $2-4 \mathrm{~cm}$ from the first incision. The samples are immediately flash-frozen in liquid nitrogen and stored at $-80^{\circ} \mathrm{C}$ for later analysis. Participants are compensated $\$ 50$ for each biopsy.

\section{Biochemical analyses}

In order to quantify mRNA expression, muscle tissue samples weighing approximately $30 \mathrm{mg}$ are homogenised from each sample. Total RNA is extracted (Qiagen RNeasy Tissue Kit, Valencia, California, USA), and the purity determined through spectrophotometry (NanoDrop ND-1000, Thermo Scientific, Waltham, Massachusetts, USA). The total RNA is reversed transcribed into cDNA using a previously published protocol. ${ }^{57}$

The primer sequences are obtained from previous reports on PGC-1 $\alpha 4^{5859}$ myostatin, FOXO3A, MAFbx and MuRF- $1,{ }^{57}$ or are designed using the Primer3 program. ${ }^{60}$ The internal control used to detect changes in gene expression during RT-PCR is GAPDH, which is based on findings from previous work examining myogenic gene expression following resistance exercise. ${ }^{59}$ Each PCR 
reaction is amplified using a Bio-Rad thermal cycler with reaction volumes and cycling protocol determined by previous work. ${ }^{5759}$

\section{Patient-reported outcomes \\ Dietary assessment}

At baseline and every week during the intervention period, all participants complete a 3-day dietary food intake that records intake over two weekdays and one weekend day. To increase compliance, all participants are monetarily compensated for each log. The dietary records are scrutinised under the supervision of a registered dietician to ensure accuracy in portion size before being entered in an online nutritional analysis application (My Fitness Pal, Under Armour, myfitnesspal.com). Daily energy consumption (kilocalories) and daily protein intake (g) are recorded for each participant. Participants who do not meet the minimum recommended daily allowance for adults of $0.8 \mathrm{~g}$ protein per kilogram of body weight are instructed to increase their daily protein intake to this level over the 1-week washout period prior to the intervention, which corresponds to the familiarisation training period. In addition, participants randomised to the RTPS and PS groups record whey PS compliance in a weekly $\log$.

\section{Physical activity assessments}

Physical activity history is assessed at baseline and post intervention using the International Physical Activity Questionnaire, which has been validated in adults over the age of $65 .{ }^{61}{ }^{62}$ In addition, all participants complete a weekly log of physical activity for any exercise performed outside the study. Participants randomised to the PS and control groups are instructed to record the home flexibility programme compliance using the physical activity $\operatorname{logs}$ and are compensated for each log. Physical activity logs are collected in person every week for RT and RTPS participants, and in person at midpoint and post intervention for PS and control participants.

\section{Quality-of-life assessments}

All quality-of-life assessments are completed in person at baseline and 12 weeks, with an investigator available to answer questions if clarification is needed. Prostate cancer-specific quality-of-life status is assessed using the Functional Assessment of Cancer Therapy Prostate (FACT-P) instrument. ${ }^{63}$ General quality-of-life status is assessed using the Short Form-36 (SF-36) ${ }^{64}$ Severity of fatigue and the impact of fatigue on activities of daily living is assessed using the Brief Fatigue Inventory (BFI) ${ }^{65}$ Emotional distress is measured using the Center for Epidemiologic Studies-Depression Scale (CES-D). ${ }^{66}$

\section{Feasibility}

The feasibility of the study is assessed through benchmarks that have been used in similar randomised controlled trials in cancer survivors and older adults, and includes (1) accrual in meeting a target enrolment of $\mathrm{n}=32$ over a 3 -year period, (2) retention of $\geq 80 \%$ in the overall sample, ${ }^{67}(3)$ exercise session adherence through attendance of at least 31 of the 36 RT sessions $(86 \%)^{68}$ and (4) PS adherence of $\geq 72 \%$ of the doses. ${ }^{45}$ In addition, tolerance of the exercise programme is assessed through adherence to the original exercises in the programme over the 3-month intervention.

\section{Data safety and monitoring}

Data are monitored by the USC NCCC Data and Safety Monitoring Committee through an annual review that includes evaluation of the current accrual relative to the planned total accrual, examination of all reported violations and review of past audits. The safety of the RTPS, RT and PS experimental arms is continuously assessed by study personnel through the absence of serious, adverse events, including extreme muscle soreness, dizziness, nausea or pain. Adverse events are identified and graded using CTCAE V.4.3 at every exercise session and testing time point and reported to research governance committees. As the study procedures and intervention were assessed to present low risk for patient safety, an interim analysis will not be performed.

\section{Sample size}

As this is a pilot study, sample size was determined based on previous studies in PCS on ADT with LBM as a primary end point, where total sample size was estimated to be 50 to detect a $1 \pm 1.25 \mathrm{~kg}$ change ${ }^{12}$ and 60 to detect a $3 \pm 3 \mathrm{~kg}$ change $^{69}$ in LBM between exercise and control groups. We estimated that seven participants per group would inform variance and effect sizes of the various outcomes for powering a future definitive RCT. As we estimated a $15 \%$ attrition rate, we aim to recruit 32 participants in total.

\section{Randomisation}

Patients are randomly assigned to RTPS, RT, PS or control by the Clinical Investigation Support Office (CISO) at USC NCCC once the patient has been contacted by the principal investigator, is found eligible and has signed the informed consent. The randomisation list was prepared in advance by a biostatistician. To prevent potential bias, study personnel do not have access to the randomisation list. Trial participants and outcome assessors are not blinded to group allocation.

\section{Data management}

Data are collected in an anonymised format on paper research charts and stored securely before being entered electronically into the secure web-based Research Electronic Data Capture application (REDCap, Vanderbilt University). Data queries are managed by a single data manager. At the end of the trial, all paper research charts will be archived securely for 5 years before being confidentially destroyed.

\section{Statistical analysis}

Intention-to-treat models will be tested using repeated measures analysis of covariance for the primary 
hypotheses that the exercise groups (RTPS + RT) exhibit greater improvements in all outcomes compared with the non-exercise groups (PS + control) from pre intervention to post intervention, adjusting for the protein factor. This will be followed by testing the secondary hypotheses that an ordinal contrast exists across groups, such that improvements are greatest in the RTPS group, followed by RT, PS and control. Missing postintervention assessments will carry the midpoint observation forward according to the intention-to-treat principle.

\section{DISCUSSION}

Previously conducted exercise investigations in PCS on ADT have been effective at enhancing muscular strength and attenuating LBM loss, but limited evidence exists for the efficacy of exercise in substantially improving sarcopenic obesity and cardiometabolic risk factors. This study incorporates several innovative approaches in countering these adverse effects of ADT including (1) employing a periodisation model as a method of structured resistance exercise progression, (2) coupling PS with resistance exercise to maximise the potential for LBM increases and (3) investigating anabolic and catabolic regulators of skeletal muscle in an attempt to define molecular mechanisms underlying an RTPS intervention in PCS.

The periodised RT programme used in this study may serve as an ideal strategy for increasing LBM, decreasing fat mass and positively altering cardiometabolic variables in PCS on ADT, as previous interventions using periodised RT in overweight, young men, ${ }^{23}$ middle-aged men $^{70}$ and elderly women ${ }^{24}$ have demonstrated concomitant improvements in all areas. Prior investigations in PCS on ADT have shown individual changes in LBM, fat mass or cardiometabolic variables, but the optimal exercise prescription to elicit improvements in all areas remains to be elucidated. In particular, LBM increases were observed in PCS on ADT following an RT intervention, but no fat loss was reported. ${ }^{21} 71$ Additionally, a combined intervention of RT+AT demonstrated beneficial changes in cardiometabolic variables, decreases in whole-body percentage of fat attenuation of LBM loss, but not LBM gain. ${ }^{9}$ These findings suggest that changes in both skeletal muscle and fat are necessary to optimise positive alterations in cardiometabolic outcomes, likely due to a complex interplay between skeletal muscle, adipose tissue and metabolism. Indeed, loss of skeletal muscle has been shown to have unfavourable effects on whole-body glucose tolerance and insulin resistance, ${ }^{72}$ and targeting the skeletal muscle-metabolism axis in PCS on ADT has been postulated to improve insulin resistance. $^{73}$ Thus, interventions that increase LBM, rather than attenuate its loss, may be most effective in improving cardiometabolic outcomes. As periodised RT has been shown to increase LBM and improve cardiometabolic variables, although in non-cancer populations, it represents a promising method to address the multiple adverse effects of ADT in PCS over aerobic training or RT+AT. Performance of the exercise programme in a controlled environment with one-to-one instruction is optimal for evaluating impact of the prescribed programme, but presents a challenge to widespread implementation. Strategies to facilitate translation of the prescribed exercise to a home-based or community-based programme will be an important subsequent step.

A second, novel aspect of this intervention is the coupling of resistance exercise with PS to maximise increases in LBM in PCS on ADT. Study participants are required to meet minimum dietary protein guidelines of at least $0.8 \mathrm{~g}$ protein $/ \mathrm{kg} /$ body weight, ${ }^{74}$ as this minimum is considered essential during all stages of cancer treatment, and may reduce the risk of cardiovascular disease. ${ }^{18}$ In addition, the $50 \mathrm{~g}$ /day PS may be beneficial by helping to meet the suggested 10\%-35\% of total calories from protein, ${ }^{18}$ as a low protein intake $(<10 \%$ total calories from protein) was associated with a higher risk of cancer and overall mortality in men over age $65 .{ }^{75}$ On the other hand, a standard $50 \mathrm{~g}$ /day supplement may be less precise than a dose normalised to body weight as previous investigations in older adults have opted for this approach. ${ }^{7677}$ Yet, similar to investigations in breast cancer survivors ${ }^{78}$ and older adults, ${ }^{45} 467980$ a standard daily amount independent of body weight was selected so that all participants would receive supplementation over their habitual dietary protein intake. Habitual protein intake and overall food consumption are monitored through 3-day diet logs over the 12-week intervention. However, no caloric control or dietary advice is implemented, which may represent a limitation in interpreting the effect of protein alone.

This study also attempts to characterise anabolic and catabolic signalling in the skeletal muscle in PCS with ADT. As loss of LBM resulting from ADT may have adverse metabolic and physical implications, understanding molecular mechanisms underlying favourable LBM changes due to exercise may contribute to potential adjunctive therapies while on ADT. In older adults and cancer patients with cachexia, the loss of LBM has been attributed to reduced signalling through the IGF-1/Akt/mTOR anabolic pathway and increased signalling through the myostatin/activin catabolic pathway. ${ }^{29}$ Resistance exercise has been shown to exert potent anabolic effects, both through the repression of myostatin and the stimulation of protein synthesis through IGF-1, ${ }^{81}$ and may therefore attenuate age-related and cancer-related impairments in muscle hypertrophy. In particular, chronic RT was shown to elevate resting levels of IGF-1 gene expression in human skeletal muscle, which was observed in association with increases in the transcriptional coactivator PGC- $1 \alpha 4 .^{58}$ In the same study, tumour-bearing mice with transgenic expression of PGC-1 $\alpha 4$ maintained LBM throughout cancer progression and exhibited lower expression of myostatin compared with control tumour-bearing mice. Collectively, these findings suggest that PGC-1 $\alpha 4$ 
may attenuate atrophy by blocking catabolic signalling through the myostatin/activin pathway via activation of IGF-1, and these findings may be observed in the context of cancer. Thus, an RTPS intervention that increases anabolic signalling through expression of PGC- $1 \alpha 4$ and IGF-1, and reduces catabolic signalling through suppression of myostatin, may reverse LBM loss in PCS despite androgen deprivation.

This study was designed to address an important insufficiency in the adjunctive care of PCS on ADT following primary therapy. As patients make the transition from treatment to survivorship, implementation of positive lifestyle behaviours is essential to reducing ADT-related detrimental effects, lowering healthcare costs for cancer and comorbidities, and enhancing health-related quality of life. This study attempts to counter the progression of sarcopenic obesity with an innovative, multilayered approach that includes a periodised model of resistance exercise, PS and investigation of molecular outcomes regulating skeletal muscle changes. Findings from this study may serve as an evidence-based exercise prescription for PCS on ADT, and inform future largescale exercise trials on parameters related to survival in patients with prostate cancer.

\section{Ethics and dissemination}

Ethics approval was obtained prior to study initiation from the USC Institutional Review Board (HS-13-00315). This trial is registered at ClinicalTrials.gov (NCT01909440). All study participants provide written informed consent prior to enrolment. Any protocol amendments will be communicated to the USC CISO NCCC and institutional review board for approval. Should a revised consent form be required, current study participants will be asked to again provide written consent. Results will be disseminated in peer-reviewed journals, national scientific conferences and cancer networks.

\section{Author affiliations}

${ }^{1}$ Division of Biokinesiology and Physical Therapy, 0strow School of Dentistry, University of Southern California, Los Angeles, California, USA

${ }^{2}$ Norris Comprehensive Cancer Center, Keck School of Medicine, University of Southern California, Los Angeles, California, USA

${ }^{3}$ Department of Preventive Medicine, Keck School of Medicine, University of Southern California, Los Angeles, California, USA

${ }^{4}$ Department of Biochemistry and Molecular Biology, Keck School of Medicine, University of Southern California, Los Angeles, California, USA

${ }^{5}$ Ellison Institute for Transformative Medicine, Keck School of Medicine, University of Southern California, Los Angeles, California, USA

${ }^{6}$ Keck School of Medicine, University of Southern California, Los Angeles, CA, USA

Contributors JLK, CMD-C, ETS, MEG and TBD: study conception and design. JLK: study coordinator, exercise trainer, endpoint assessments and data manager. JLK and CMD-C: study management. TBD and MEG: study physicians and medical advice. JLK, ETS and GJS: exercise intervention and performance testing conception. JLK and TBD: biospecimen collection. JLK and CJL: statistical analyses. JLK, CMD-C, JRC and MEG: conception and biospecimen analyses. JLK drafted and finalised the manuscript. All authors have read and approved the final manuscript.

Funding This pilot trial was supported by doctoral grants from the National Strength and Conditioning Association and the California State University Chancellor's Doctoral Incentive Program.

Competing interests None declared.
Ethics approval University of Southern California Institutional Review Board.

Provenance and peer review Not commissioned; externally peer reviewed.

Open Access This is an Open Access article distributed in accordance with the Creative Commons Attribution Non Commercial (CC BY-NC 4.0) license, which permits others to distribute, remix, adapt, build upon this work non-commercially, and license their derivative works on different terms, provided the original work is properly cited and the use is non-commercial. See: http://creativecommons.org/ licenses/by-nc/4.0/

(C) Article author(s) (or their employer(s) unless otherwise stated in the text of the article) 2017. All rights reserved. No commercial use is permitted unless otherwise expressly granted.

\section{REFERENCES}

1. Siegel RL, Miller KD, Jemal A, et al. CA: a Cancer journal for clinicians. 2015;651:5-29.

2. Levine GN, D'Amico AV, Berger P, et al. Androgen-deprivation therapy in prostate Cancer and cardiovascular risk: a science advisory from the american Heart Association, American Cancer society, and american Urological Association: endorsed by the American Society for Radiation Oncology. CA: a cancer journal for clinicians 2010;603:194-201.

3. Alberti KG, Zimmet P, Shaw J. Metabolic syndrome--a new worldwide definition. A Consensus Statement from the International Diabetes Federation. Diabet Med 2006;23:469-80.

4. Conteduca V, Di Lorenzo G, Bozza G, et al. Metabolic syndrome as a peculiar target for management of prostate cancer patients. Clin Genitourin Cancer 2013;11:211-20.

5. Galvão DA, Taaffe DR, Spry N, et al. Reduced muscle strength and functional performance in men with prostate cancer undergoing androgen suppression: a comprehensive cross-sectional investigation. Prostate Cancer Prostatic Dis 2009;12:198-203.

6. Stokes ME, Ishak J, Proskorovsky I, et al. Lifetime economic burden of prostate cancer. BMC Health Serv Res 2011;11:349.

7. Roberts CK, Hevener AL, Barnard RJ. Metabolic syndrome and insulin resistance: underlying causes and modification by exercise training. Compr Physiol 2013;3:1-58.

8. Bourke L, Doll H, Crank H, et al. Lifestyle intervention in men with advanced prostate cancer receiving androgen suppression therapy: a feasibility study. Cancer Epidemiol Biomarkers Prev 2011;20:647-57.

9. Cormie P, Galvão DA, Spry N, et al. Can supervised exercise prevent treatment toxicity in patients with prostate cancer initiating androgen-deprivation therapy: a randomised controlled trial. BJU Int 2015;115:256-66.

10. Culos-Reed SN, Robinson JW, Lau H, et al. Physical activity for men receiving androgen deprivation therapy for prostate cancer: benefits from a 16-week intervention. Support Care Cancer 2010;18:591-9.

11. Galvão DA, Spry N, Denham J, et al. A Multicentre year-long randomised controlled trial of exercise training targeting physical functioning in men with prostate cancer previously treated with androgen suppression and radiation from TROG 03.04 RADAR. Eur Urol 2014;65:856-64.

12. Galvão DA, Taaffe DR, Spry N, et al. Combined resistance and aerobic exercise program reverses muscle loss in men undergoing androgen suppression therapy for prostate cancer without bone metastases: a randomized controlled trial. J Clin Oncol 2010;28:340-7.

13. Segal RJ, Reid RD, Courneya KS, et al. Resistance exercise in men receiving androgen deprivation therapy for prostate cancer. $J$ Clin Clinical Oncology 2003;21:1653-9.

14. Bourke L, Gilbert S, Hooper R, et al. Lifestyle changes for improving disease-specific quality of life in sedentary men on long-term androgen-deprivation therapy for advanced prostate cancer: a randomised controlled trial. Eur Urol 2014;65:865-72.

15. Hvid T, Winding K, Rinnov A, et al. Endurance training improves insulin sensitivity and body composition in prostate cancer patients treated with androgen deprivation therapy. Endocr Relat Cancer 2013;20:621-32.

16. Santa Mina D, Alibhai S MH, Matthew AG, et al. A randomized trial of aerobic versus resistance exercise in prostate Cancer survivors. J Aging Phys Act 2013;21:455-78.

17. In: Winters-Stone KM, Dieckmann N, Maddalozzo GF, ResistanceExercise Reduces Body Fat and Insulin During AndrogenDeprivation Therapy forProstate Cancer. Oncology nursing forum, 2015. 
18. Rock CL, Doyle C, Demark-Wahnefried W, et al. Nutrition and physical activity guidelines for cancer survivors. CA: A Cancer Journal for Clinicians 2012;62:242-74.

19. Schmitz KH, Courneya KS, Matthews C, et al. American College of Sports Medicine roundtable on exercise guidelines for cancer survivors. Med Sci Sports Exerc 2010;42:1409-26.

20. Galvão DA, Nosaka K, Taaffe DR, et al. Resistance training and reduction of treatment side effects in prostate cancer patients. Med Sci Sports Exerc 2006;38:2045-52.

21. Nilsen TS, Raastad T, Skovlund E, et al. Effects of strength training on body composition, physical functioning, and quality of life in prostate cancer patients during androgen deprivation therapy. Acta Oncol 2015;54:1805-13.

22. Haff GG. Roundtable Discussion: periodization of Training-Part 1. Strength \& Conditioning Journal 2004;261:50-69.

23. Ahmadizad S, Ghorbani S, Ghasemikaram M, et al. Effects of shortterm nonperiodized, linear periodized and daily undulating periodized resistance training on plasma adiponectin, leptin and insulin resistance. Clin Biochem 2014;47:417-22.

24. Botero JP, Shiguemoto GE, Prestes J, et al. Effects of long-term periodized resistance training on body composition, leptin, resistin and muscle strength in elderly post-menopausal women. $J$ Sports Med Phys Fitness 2013;53:289-94.

25. Turner A. The Science and Practice of Periodization: a brief review. Strength Cond J 2011;33:34-46.

26. Fry CS, Drummond MJ, Glynn EL, et al. Aging impairs contractioninduced human skeletal muscle mTORC1 signaling and protein synthesis. Skelet Muscle 2011;11:1.

27. Hulmi JJ, Lockwood CM, Stout JR. Effect of protein/essential amino acids and resistance training on skeletal muscle hypertrophy: a case for whey protein. Nutr Metab 2010;7:51.

28. Finger D, Goltz FR, Umpierre D, et al. Effects of protein supplementation in older adults undergoing resistance training: a systematic review and meta-analysis. Sports Med 2015;45:245-55

29. Fearon KC, Glass DJ, Guttridge DC. Cancer cachexia: mediators, signaling, and metabolic pathways. Cell Metab 2012;16:153-66.

30. Hameed M, Lange KH, Andersen JL, et al. The effect of recombinant human growth hormone and resistance training on IGF-I mRNA expression in the muscles of elderly men. J Physio/ 2004;555:231-40.

31. Hulmi JJ, Ahtiainen JP, Kaasalainen T, et al. Postexercise myostatin and activin Ilb mRNA levels: effects of strength training. Med Sci Sports Exerc 2007;39:289.

32. Psilander N, Damsgaard R, Pilegaard H. Resistance exercise alters MRF and IGF-I mRNA content in human skeletal muscle. J Appl Physiol 2003;95:1038-44.

33. Ss H, Dhaliwal SS, Hills AP, et al. 2 weeks of aerobic, resistance or combination exercise training on cardiovascular risk factors in the overweight and obese in a randomized trial. BMC public health 2012;121:1

34. Campos GE, Luecke TJ, Wendeln HK, et al. Muscular adaptations in response to three different resistance-training regimens: specificity of repetition maximum training zones. Eur J Appl Physiol 2002;88:50-60.

35. Schoenfeld BJ. Potential mechanisms for a role of metabolic stress in hypertrophic adaptations to resistance training. Sports Med 2013;43:179-94.

36. Kraemer WJ, Ratamess NA. Fundamentals of resistance training: progression and exercise prescription. Med Sci Sports Exerc 2004;36:674-88.

37. Akuthota V, Nadler SF. Core strengthening. Arch Phys Med Rehabil 2004;85(3 Suppl 1):S86-92.

38. Lehman GJ. Resistance training for performance and injury prevention in golf. J Can Chiropr Assoc 2006;50:27-42.

39. Myer GD, Ford KR, Palumbo JP, et al. Neuromuscular training improves performance and lower-extremity biomechanics in female athletes. J Strength Cond Res 2005;19:51-60.

40. Hagins M, Adler K, Cash M, et al. Effects of practice on the ability to perform lumbar stabilization exercises. J Orthop Sports Phys Ther 1999;29:546-55.

41. Herman SL, Smith DT. Four-week dynamic stretching warm-up intervention elicits longer-term performance benefits. J Strength Cond Res 2008;22:1286-97.

42. Winters-Stone KM, Dobek JC, Bennett JA, et al. Skeletal response to resistance and impact training in prostate cancer survivors. Med Sci Sports Exerc 2014;46:1482-8.

43. Galvão DA, Taaffe DR, Cormie P, et al. Efficacy and safety of a modular multi-modal exercise program in prostate cancer patients with bone metastases: a randomized controlled trial. BMC Cancer 2011;11:517.

44. Katsanos CS, Chinkes DL, Paddon-Jones D, et al. Whey protein ingestion in elderly persons results in greater muscle protein accrual than ingestion of its constituent essential amino acid content. Nutr Res 2008;28:651-8.

45. Chalé A, Cloutier GJ, Hau C, et al. Efficacy of whey protein supplementation on resistance exercise-induced changes in lean mass, muscle strength, and physical function in mobility-limited older adults. J Gerontol A Biol Sci Med Sci 2013;68:682-90.

46. Verdijk LB, Jonkers RA, Gleeson BG, et al. Protein supplementation before and after exercise does not further augment skeletal muscle hypertrophy after resistance training in elderly men. Am J Clin Nutr 2009;89:608-16.

47. Hart NH, Nimphius S, Spiteri T, et al. Segmental musculoskeletal examinations using Dual-Energy X-Ray Absorptiometry (DXA): Positioning and analysis considerations. J Sports Sci Med 2015;14:620.

48. Baumgartner RN. Body composition in healthy aging. Ann N Y Acad Sci 2000;904:437-48.

49. Baumgartner RN, Stauber PM, McHugh D, et al. Cross-sectional age differences in body composition in persons $60+$ years of age. $J$ Gerontol A Biol Sci Med Sci 1995;50:M307-M316.

50. Baumgartner RN, Koehler KM, Gallagher D, et al. Epidemiology of Sarcopenia among the elderly in New Mexico. Am J Epidemiol 1998;147:755-63.

51. Batsis JA, Barre LK, Mackenzie TA, et al. Variation in the prevalence of Sarcopenia and sarcopenic obesity in older adults associated with different research definitions: dual-energy X-ray absorptiometry data from the National Health and Nutrition Examination Survey 19992004. J Am Geriatr Soc 2013;61:974-80.

52. Reynolds JM, Gordon TJ, Robergs RA. Prediction of one repetition maximum strength from multiple repetition maximum testing and anthropometry. J Strength Cond Res 2006;20:584-92.

53. Knutzen KM, Brilla LR, Caine D. Validity of 1RM Prediction Equations for Older Adults. J Strength Cond Res 1999;13:242-6.

54. Brzycki M. Strength Testing-Predicting a One-Rep Max from Repsto-Fatigue. J Phys Educ Recreat Dance 1993;641:88-90.

55. Soubeyran P, Fonck M, Blanc-Bisson C, et al. Predictors of early death risk in older patients treated with First-Line Chemotherapy for Cancer. Journal of Clinical Oncology 2012;30:1829-34.

56. Gribble PA, Hertel J, Plisky P. Using the Star Excursion Balance Test to assess Dynamic Postural-Control Deficits and Outcomes in Lower Extremity Injury: a Literature and Systematic Review. J Athl Train 2012;47:339-57.

57. Dieli-Conwright CM, Spektor TM, Rice JC, et al. Influence of hormone replacement therapy on eccentric exercise induced myogenic gene expression in postmenopausal women. $J$ Appl Physiol 2009;107:1381-8.

58. Ruas JL, White JP, Rao RR, et al. A PGC- $1 \alpha$ isoform induced by resistance training regulates skeletal muscle hypertrophy. Cell 2012;151:1319-31.

59. Dieli-Conwright CM, Kiwata JL, Tuzon CT, et al. Acute response of PGC-1 $\alpha$ and IGF- 1 isoforms to maximal eccentric exercise in skeletal muscle of Postmenopausal Women. J Strength Cond Res 2016;30:1161-70.

60. Untergasser A, Cutcutache I, Koressaar T, et al. Primer3--new capabilities and interfaces. Nucleic Acids Res 2012;40:e115.

61. Hurtig-Wennlöf A, Hagströmer M, Olsson LA. The International Physical Activity Questionnaire modified for the elderly: aspects of validity and feasibility. Public Health Nutr 2010;13:1311-54.

62. Tomioka K, Iwamoto J, Saeki K, et al. Reliability and validity of the International Physical Activity Questionnaire (IPAQ) in elderly adults: the Fujiwara-kyo study. J Epidemiol 2011;21:459-65.

63. ACoS M. ACSM's Guide to Exercise and Cancer Survivorship. Champaign: IL Human Kinetics, 2012.

64. Ware JE, Gandek B. Overview of the SF-36 Health Survey and the International Quality of Life Assessment (IQOLA) Project. J Clin Epidemiol 1998:51:903-12.

65. Mendoza TR, Wang XS, Cleeland CS, et al. The rapid assessment of fatigue severity in Cancer patients: use of the brief fatigue inventory. Cancer 1999;85:1186-96.

66. Radloff LS. The CES-D Scale: A Self-Report Depression Scale for Research in the General Population. Appl Psychol Meas 1977;1:385-401.

67. Demark-Wahnefried W, Nix JW, Hunter GR, et al. Feasibility outcomes of a presurgical randomized controlled trial exploring the impact of caloric restriction and increased physical activity versus a wait-list control on tumor characteristics and circulating biomarkers in men electing prostatectomy for prostate Cancer. BMC Cancer 2016;16:161:1.

68. Segal RJ, Reid RD, Courneya KS, et al. Randomized Controlled Trial of Resistance or Aerobic Exercise in Men Receiving Radiation therapy for Prostate Cancer. Journal of Clinical Oncology 2009;27:344-51. 
69. Thorsen L, Nilsen TS, Raastad T, et al. A randomized controlled trial on the effectiveness of strength training on clinical and muscle cellular outcomes in patients with prostate cancer during androgen deprivation therapy: rationale and design. BMC Cancer 2012;12:123.

70. Augusto Libardi C, Bonganha V, Soares Conceição M, et al. The periodized resistance training promotes similar changes in lipid profile in middle-aged men and women. J Sports Med Phys Fitness 2012;52:286-92.

71. Cormie P, Newton RU, Spry N, et al. Safety and efficacy of resistance exercise in prostate Cancer patients with bone metastases. Prostate Cancer Prostatic Dis 2013;16:328-35.

72. Febbraio MA, Hiscock N, Sacchetti M, et al. Interleukin-6 is a novel factor mediating glucose homeostasis during skeletal muscle contraction. Diabetes 2004;53:1643-8.

73. Basaria S, Bhasin S. Targeting the skeletal muscle-metabolism Axis in prostate-cancer therapy. N Engl J Med 2012;367:965-7.

74. Lichtenstein AH, Appel LJ, Brands M, et al. Diet and lifestyle recommendations revision 2006: a scientific statement from the american Heart Association Nutrition Committee. Circulation 2006;114:82-96.

75. Levine ME, Suarez JA, Brandhorst S, et al. Low protein intake is associated with a Major reduction in IGF-1, Cancer, and overall mortality in the 65 and Younger but not older Population. Cell Metab 2014;19:407-17.

76. Campbell WW, Crim MC, Young VR, et al. Effects of resistance training and dietary protein intake on protein metabolism in older adults. Am J Physiol 1995;268:E1143-E53.

77. Candow DG, Chilibeck PD, Facci M, et al. Protein supplementation before and after resistance training in older men. Eur J Appl Physiol 2006;97:548-56

78. Madzima TA, Ormsbee MJ, Schleicher EA, et al. Effects of resistance training and protein supplementation in breast Cancer survivors. Med Sci Sports Exerc 2017;49:1283-1292-92.

79. Leenders M, Verdijk LB, Van der Hoeven L, et al. Protein supplementation during resistance-type exercise training in the elderly. Med Sci Sports Exerc 2013;45:542-52.

80. Tieland M, Dirks ML, van der Zwaluw N, et al. Protein supplementation increases muscle mass gain during prolonged resistance-type exercise training in frail elderly people: a randomized, double-blind, placebo-controlled trial. J Am Med Dir Assoc 2012;13:713-9.

81. Bowen TS, Schuler G, Adams V. Skeletal muscle wasting in cachexia and Sarcopenia: molecular pathophysiology and impact of exercise training. Journal ofcachexia, sarcopenia and muscle 2015;63:197-207. 